\title{
Towards understanding the gliotoxin detoxification mechanism: in vivo thiomethylation protects yeast from gliotoxin cytotoxicity
}

\author{
Elizabeth B. Smith, Stephen K. Dolan, David A. Fitzpatrick, Sean Doyle and Gary W. Jones* \\ Department of Biology, Maynooth University, Maynooth, County Kildare, Ireland. \\ * Corresponding Author: \\ Gary Jones, Tel: +353 1708 3839; Fax: +353 1708 3845; E-mail: gary.jones@nuim.ie
}

\begin{abstract}
Gliotoxin (GT) is a mycotoxin produced by some species of ascomycete fungi including the opportunistic human pathogen Aspergillus fumigatus. In order to produce GT the host organism needs to have evolved a selfprotection mechanism. GT contains a redox-cycling disulfide bridge that is important in mediating toxicity. Recently is has been demonstrated that $A$. fumigatus possesses a novel thiomethyltransferase protein called GtmA that has the ability to thiomethylate GT in vivo, which aids the organism in regulating GT biosynthesis. It has been suggested that thiomethylation of GT and similar sulfur-containing toxins may play a role in providing self-protection in host organisms. In this work we have engineered Saccharomyces cerevisiae, a GT-naïve organism, to express $A$. fumigatus GtmA. We demonstrate that GtmA can readily thiomethylate GT in yeast, which results in protection of the organism from exogenous GT. Our work has implications for understanding the evolution of GT self-protection mechanisms in organisms that are GT producers and non-producers.
\end{abstract}

doi: $10.15698 / \mathrm{mic} 2016.03 .485$ Received originally: 09.09.2015; in revised form: 22.12.2015, Accepted 07.01.2016, Published 19.02.2016.

Keywords: gliotoxin, Aspergillus fumigatus, Saccharomyces cerevisiae, oxidoreductase GliT, thiomethyltransferase GtmA.

Abbreviations:
BmGT - bisdethiobis(methylthio)-
gliotoxin,
ETP - epipolythiodioxopiperazine,
GSH - glutathione,
GT - gliotoxin,
GT-(SH) - reduced dithiol form of
gliotoxin.

Abbreviations:

gliotoxin,

ETP - epipolythiodioxopiperazine, tathione,

GT-(SH) $)_{2}$ - reduced dithiol form of

\section{INTRODUCTION}

Gliotoxin (GT) is a fungal natural product with known antibiotic and antifungal properties and is biosynthesized by the opportunistic human pathogen, Aspergillus fumigatus, as well as by related ascomycetes [1-3]. Gliotoxin biosynthesis is encoded by the gli gene cluster, which contains 13 genes, and the function of most of these genes has now been elucidated [4]. Effectively, GT is the prototypic epipolythiodioxopiperazine (ETP) and is structurally and functionally related to a range of disulfide bridgecontaining non-ribosomal peptides including, amongst others, sporidesmin A and sirodesmin $[2,5]$. GT is redoxactive; thus, cycling between the oxidized and reduced dithiol form (GT-(SH) $)_{2}$ ) can generate reactive oxygen species, which have deleterious cellular effects. GT autoinduces its own biosynthesis by activating and maintaining gli cluster expression [6]. In animal cells, GT can inactivate selected protein functionality by covalent interaction with protein thiols and also deplete cellular glutathione (GSH) [7, 8]. Indeed, the cytotoxicity of GT is such that an independently regulated gene within the gli cluster, gliT encodes GT oxidoreductase which recognizes GT-(SH) ${ }_{2}$ and catalyzes disulfide bridge closure and is essential in $A$. $f u-$ migatus for self-protection against exogenous GT [9-11].

GT is not biosynthesized by Saccharomyces cerevisiae (baker's yeast). As a consequence, no endogenous protection system against GT exists in this species and $S$. cerevisiae growth can be inhibited by exposure to GT [10, 12, 13]. Expression of the $A$. fumigatus gliT-mediated selfprotection system in yeast has been shown to confer GT resistance, as has deletion of GSH1, responsible for GSH biosynthesis [10, 12]. Thus, by enabling disulfide bridge closure, preventing the GSH-mediated chemical reduction of exogenously-added GT to GT-(SH) ${ }_{2}$ or facilitating GT efflux, the resistance of gliotoxin-naïve species to this redoxactive molecular species can be augmented.

Gliotoxin bis-thiomethyltransferase (GtmA), the first bis thiomethyltransferase so far identified in any species, has recently been described in $A$. fumigatus and it has been shown to convert GT-(SH) ${ }_{2}$ to bisdethiobis(methylthio)gliotoxin (BmGT) (Figure 1) [14]. GtmA has also been contemporaneously characterized by others, where it is referred to as TmtA [15]. Interestingly, although GtmA is encoded outside the gli cluster, its expression is 
induced by GT and it has been convincingly demonstrated to dissipate GT levels thereby attenuating gli cluster activity and concomitant GT biosynthesis [14]. In effect, GtmA appears to be the 'off-switch' for GT biosynthesis in A. fumigatus. Although an unidentified thiomethyltransferase has been shown to confer resistance to a bacterial nonribosomal peptide, holomycin, in Streptomyces clavivulgaris [16], gtmA deletion has effectively no impact on the sensitivity of $A$. fumigatus to $\mathrm{GT}[14,15]$.

Expression of gtmA in S. cerevisiae was therefore undertaken to further investigate the functionality of this enzyme and to explore the hypothesis that, in the absence of concomitant GT biosynthesis and a gliT-mediated selfprotection system, GtmA could confer resistance to GT via BmGT formation.

\section{RESULTS AND DISCUSSION}

Expression of $\operatorname{gtm} A$ in yeast increases resistance to gliotoxin

While it has been clearly demonstrated that GliT provides the primary self-protection mechanism against GT in $A$. fumigatus $[9,10]$ and that the production of $B m G T$ via GtmA is primarily linked to GT biosynthesis and regulation [14], given that the production of BmGT will nullify GT ability to redox cycle, we hypothesized that under certain biological conditions GtmA may be able to provide direct protection against GT toxicity. In previous work from our group and others $[10,12,13]$ yeast was utilized as a tool to explore the in vivo effects of exposure to GT. Following cloning of gtmA under control of the constitutive SSA2 promoter we demonstrated that $\operatorname{gtm} A$ is well expressed in yeast (Figure 2A). Growth of yeast is severely impaired on medium containing gliotoxin ( 8 and $16 \mu \mathrm{g} / \mathrm{mL}$, respectively) but expression of gtmA in yeast provides protection against this cytotoxicity (Figure $2 \mathrm{~B}$ ). The level of protection provided to yeast by gtmA is more prominent than that provided by gliT expression (Figure $2 \mathrm{~B}$ ). Additionally, the level of protection provided when both gliT and gtmA are expressed in yeast is comparable to gtmA expression alone,

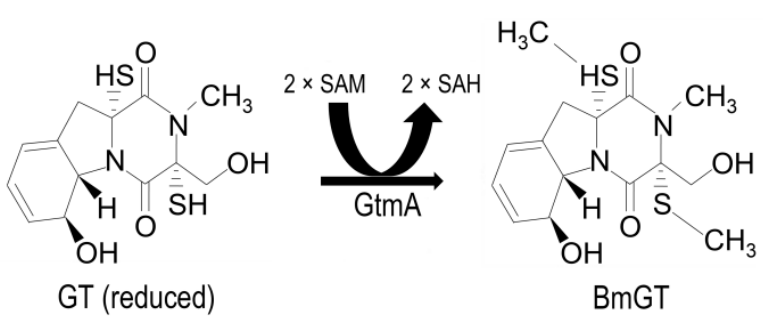

FIGURE 1: Conversion of reduced GT to BmGT is mediated by GtmA protein. Reduced GT undergoes bis-thiomethylation via the action of GtmA protein and requires two molecules of SAM for the reaction to reach completion [14]. Consequently two molecules of SAH are produced that re-enter the methyl-methionine cycle [17].

which reflects the apparent more efficient GT detoxification of GtmA compared to GliT when engineered in yeast. The expression of both gtmA and glit does not allow yeast to efficiently grow on GT concentration above those used in this study [data not show]. Expression of the other methyltransferases encoded within the gli-cluster, glim and gliN [15], did not provide any protection in yeast against GT toxicity (data not shown).

\section{Expression of gtmA in yeast causes BmGT formation from exogenously-added gliotoxin}

Yeast does not contain an ortholog or homolog of $A$. fumigatus gtmA [14]. Consequently it is not surprising that we have never detected the production of BmGT in yeast following exposure to GT (Figure $3 \mathrm{~A}$ and Figure $4 \mathrm{~A}$ ). The expression of gtmA in yeast provides this GT-naïve organism with the capability of converting GT to BmGT (Figure $3 \mathrm{~B})$. The production of BmGT in yeast expressing $\mathrm{gtmA}$ is as a result of utilizing exogenous GT as a substrate (Figure 4B).

\section{Biological relevance for gliotoxin conversion to BmGT}

What is the biological significance for GT to BmGT conver-

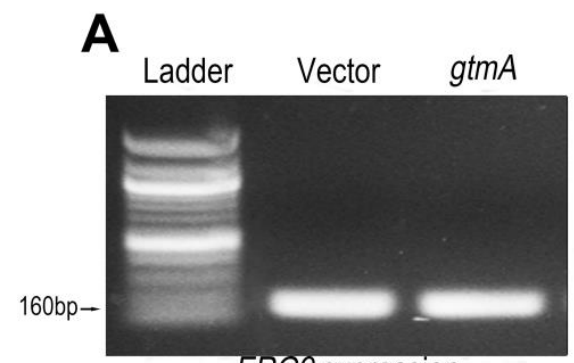

ERG9 expression

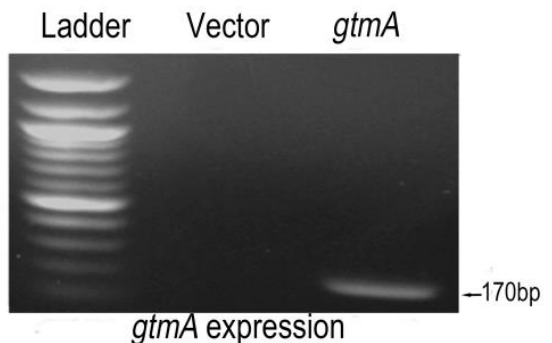

gtmA expression
FIGURE 2: Expression of gtmA in yeast protects against GT cytotoxicity. (A) RT-PCR demonstrating that gtmA mRNA is stably expressed in yeast. Yeast ERG9 mRNA production is shown as a positive control. (B) Expression of $A$. fumigatus genes gtmA or gliT in yeast provides protection against GT cytotoxicity.

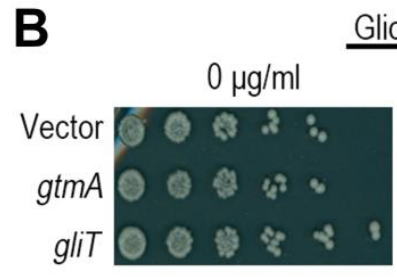

Gliotoxin concentration

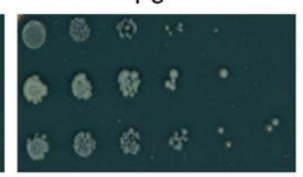

$16 \mu \mathrm{g} / \mathrm{ml}$

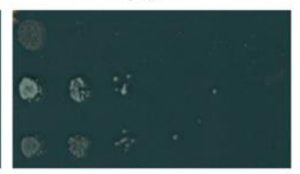


sion? The results we present here clearly show that GT conversion to BmGT protects against cytotoxicity and therefore appear to support the suggestion that thiomethylation of GT is a protection mechanism, perhaps from exogenous and/or endogenously produced GT. However, to uncover the true biological relevance of GT to BmGT conversion we need to consider this reaction in the context of organisms that produce GT and those that do not. Recent evidence has clearly shown that deletion of $\operatorname{gtm} A$, and hence the removal of the ability to convert GT to BmGT, in A. fumigatus does not produce a GT-sensitive phenotype $[14,15]$. This is due to the maintenance of a fully functional, primary detoxification system, GliT, in a gtmA deletion strain. Furthermore, it has recently been demonstrated that GliT functions in conjunction with the GT-specific transporter GliA to maintain intracellular GT at a level that is not toxic to the host cell [18]. The production of $\mathrm{BmGT}$ in
A. fumigatus facilitates the secretion of this product in a GliA independent manner [18]. The secretion of BmGT from fungal cells most likely occurs through a non-specific mechanism as the molecule is efficiently secreted from yeast (this study) and from GliA-deficient $A$. fumigatus [18]. Thus the primary physiological relevance of converting GT to BmGT in GT-producing organisms appears to be as a negative regulator of GT biosynthesis, followed by secretion of the inactive thiomethylated derivative [18]. However, the in vivo consequence of GtmA activity, and consequent BmGT production and secretion, will depend upon whether an organism is a GT producer or not.

Here we clearly show that engineering a GT to BmGT conversion mechanism into a GT naïve organism, such as yeast, can provide efficient protection against exogenous GT (Figures 2, 3 and 4). The yeast genome does not encode for any GtmA homologs and does not endogenously con-
A

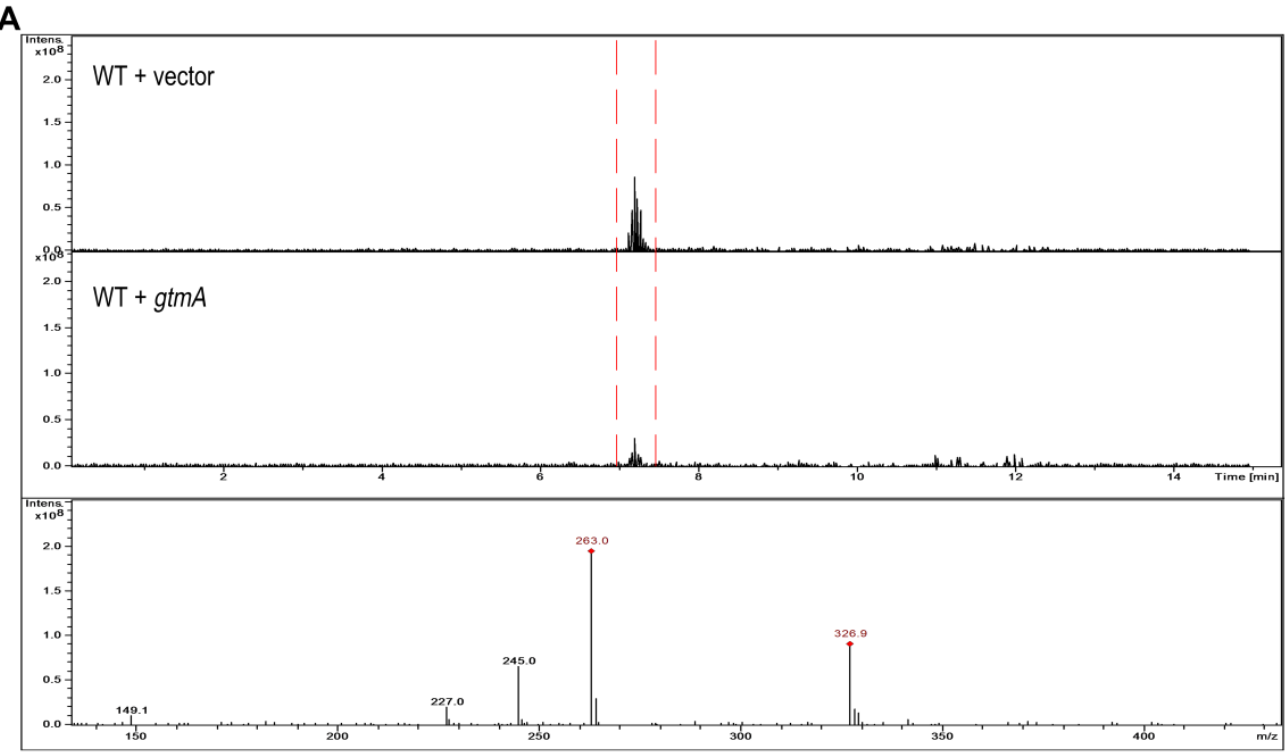

B

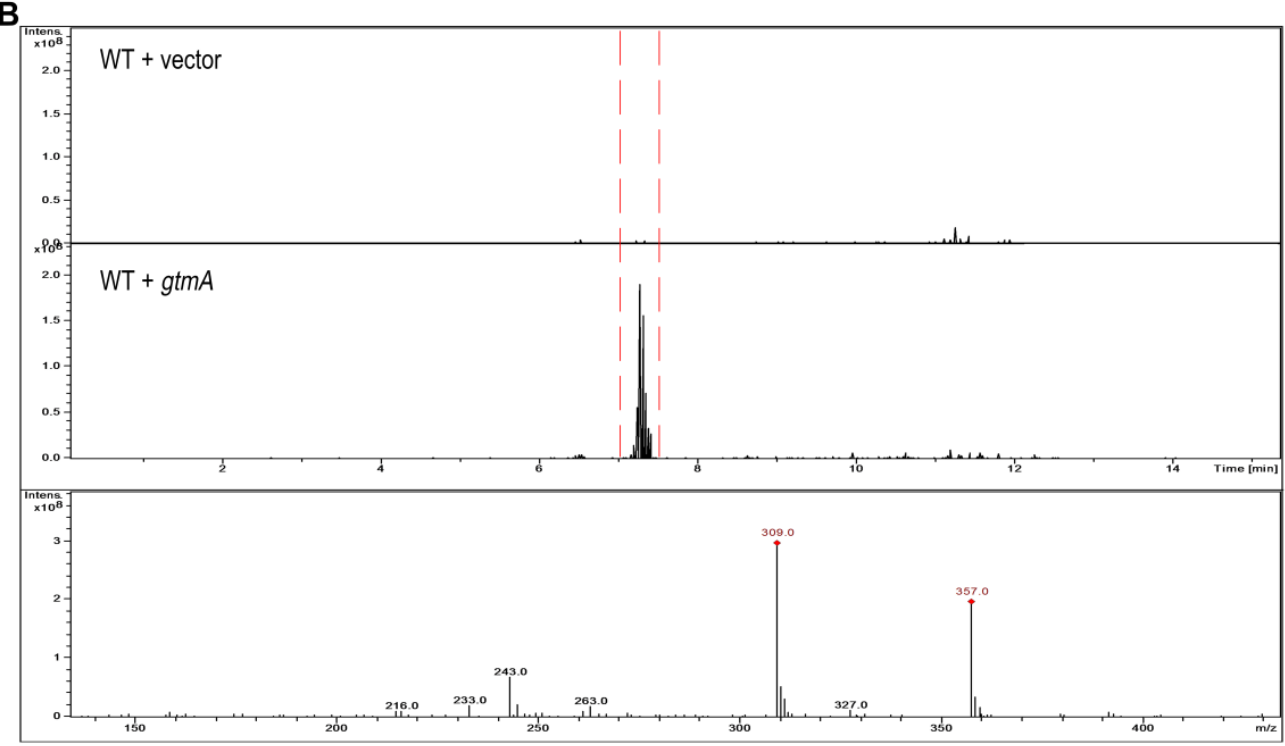

FIGURE 3: Detection and monitoring of GT and BmGT in yeast using mass spectrometry. (A) GT is readily detected in supernatants of yeast cells exposed to exogenous GT. (B) BmGT can only be detected in supernatants from yeast cells, which express gtmA, following exposure to exogenous GT. 
A

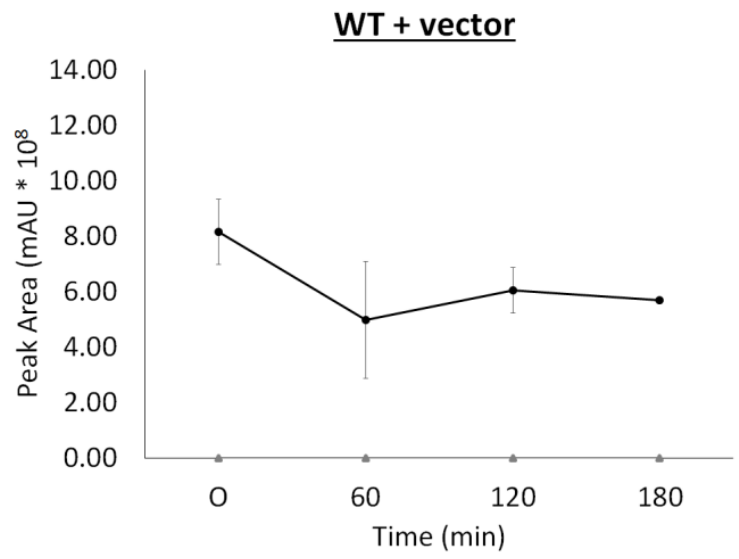

B

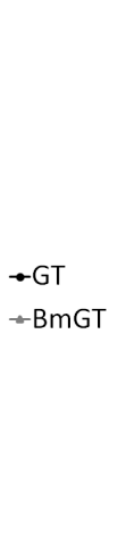

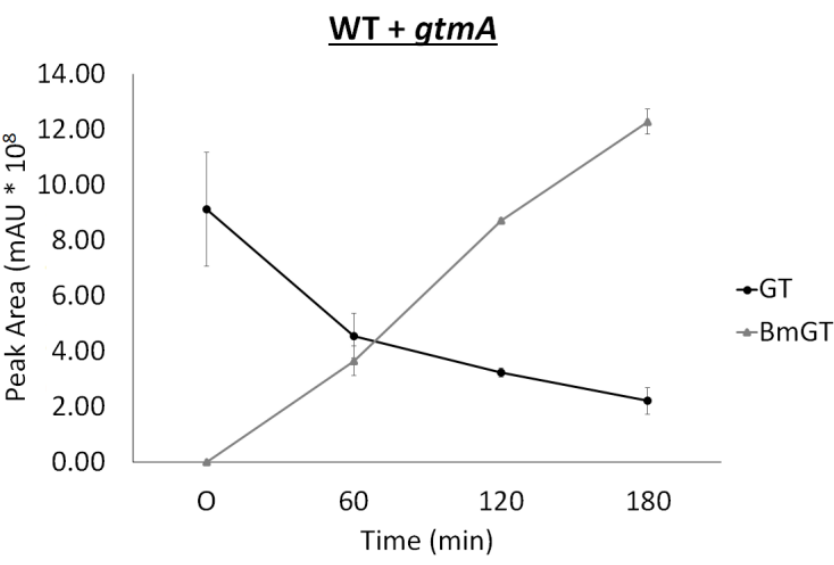

FIGURE 4: Conversion of GT to BmGT over time in yeast expressing GtmA. (A) BmGT is not detected in supernatants of yeast cells exposed to exogenous GT over $3 \mathrm{~h}$ exposure. (B) BmGT is readily detected in supernatants from yeast cells expressing gtmA. Increased levels of BmGT can be detected over a 3-hour time period and correlate with a diminution GT levels.

vert GT to BmGT (Figure 3). Previous phylogenetic analysis identified organisms within the fungal kingdom that possess GtmA homologs [14] and the implications from the results of this study suggest that a GT non-producing organism that possesses a GtmA homolog may well utilize such a protein as a defense mechanism against GT exposure. The absence of GT production in such organisms may suggest that from an evolutionary perspective such GtmA homologs may have evolved to carry out other GTindependent functions in the cell and, if they possess the ability to produce BmGT, this may not be the primary function of the protein. Conversely, GT non-producing fungi may have retained GtmA homologs as a means of occupying the same habitats as GT-producing fungi.

Moreover, the possibility does exist that GtmA homologs in GT naïve fungi constitute an ancient ETP genericdefense mechanism that has allowed the acquisition and development of the GT-producing gene cluster, or other ETP clusters. The evolution of the primary detoxification system involving GliT and GliA, in conjunction with the presence of GtmA, has then allowed for intricate systemslevel interactions to develop in organisms such as $A$. $f u$ migatus, as recently shown for GT biosynthesis and interplay with the methyl/methionine cycle [18] and for regulation of the gli-cluster itself [14]. Any effect upon GT sensitivity of removing the GT to BmGT conversion system in $A$. fumigatus, or any GT-producing organism, may only become apparent when undertaken along with the concomitant abrogation of the primary GT-protection mechanism from the organism. Support for this hypothesis comes from the recent discovery that deletion of the gtmA homolog in GT-naïve Aspergillus niger results in sensitivity to exogenously added GT [19].

\section{MATERIALS AND METHODS}

\section{Yeast strains, plasmids and genetic methods}

The $S$. cerevisiae strain used in this study was BY4741 (MATa; his $3 \Delta$ 1; leu2 $\Delta 0$; met $15 \Delta 0$; ura3 $\Delta 0$ ) and was obtained from Euroscarf. All media used were as previously described by [20]. Cultures were grown at $30^{\circ} \mathrm{C}$ with shaking at $200 \mathrm{rpm}$.

To observe the effects of gtmA expression in S. cerevisiae, gtmA was amplified from A. fumigatus CDNA (ATCC26933) using primers gtmA $F$ and gtmA $R$ (Table 1 ) and cloned into the yeast shuttle vector pC210 as previously described by [10]. Briefly, primers were designed that incorporated $\mathrm{Ndel}$ and $\mathrm{Sph} /$ restriction sites. Following PCR amplification of the gtmA gene, digestion of the gtmA PCR product and of $\mathrm{pC} 210$ was carried out followed by ligation using T4 DNA ligases (Promega) according to manufacturer's instructions to create pC210-gtmA. Cloning of the gtmA fragment and construct were confirmed using Sanger sequencing.

Growth analysis was carried out by diluting an overnight culture of cells in fresh medium lacking leucine (-LEU) to an $\mathrm{OD}_{600}=0.2$ and incubated at $30^{\circ} \mathrm{C}$ shaking $200 \mathrm{rpm}$ till an $\mathrm{OD}_{600}=0.4$ was reached. Cells were transferred to a microtiter

TABLE 1. Oligonucleotide primers used in this study.

\begin{tabular}{ll}
\hline Primer Name & Sequence 5'-3' $^{\prime}$ \\
\hline gtmA F & $\begin{array}{l}\text { AAAAAACATATGATGTCCAAGTCAGAC- } \\
\text { TACATCCAG }\end{array}$ \\
\hline gtmA R & $\begin{array}{l}\text { AAAAAAGCATGCCTAGGGCTT- } \\
\text { GAGGCCGGTTG }\end{array}$ \\
\hline ERG9 INTERAL F & GACTTATTTGGCCGGTATCCACG \\
\hline ERG9 INTERNAL R & CTCGACACAGCCACGCAAAGTCC \\
\hline gtmA RT-PCR F & TCCAGCGTACTCAACCACAC \\
\hline gtmA RT-PCR R & CGTCTGGAAAGCTCTGGAA \\
\hline
\end{tabular}


plate and serially diluted. Cells were transferred to -LEU plates containing the desired concentration of GT (Sigma) using a replicator and incubated at $30^{\circ} \mathrm{C}$ for $48 \mathrm{~h}$, with further monitoring at room temperature for $72 \mathrm{~h}$.

\section{RT-PCR}

Total RNA was extracted from $5 \mathrm{~mL}$ cultures of the yeast strains grown overnight at $30^{\circ} \mathrm{C}$ shaking $200 \mathrm{rpm}$. RNA was extracted using the QIAGEN RNeasy plant mini kit as per manufacturers' guidelines. RNA was DNase treated using DNase I kit (Sigma-Aldrich), according to the manufacturer's recommendations. RNA concentrations were measured using a Nano Drop 1000 Spectrophotometer (Mason Technology).

cDNA was synthesized using the qScript ${ }^{\mathrm{TM}}$ CDNA Supermix (Quanta Biosciences) as per manufacturer's instructions. AccuTaq LA polymerase (Sigma) was used to amplify a housekeeping gene ERG9 as a control and the gene of interest gtmA. The primers used in RT-PCR reactions are listed in Table 1. PCR reactions were conducted in a PTC-200 Peltier Thermal Cycler (MJ Research) and the program consisted of 1 cycle of $95^{\circ} \mathrm{C}$ for $5 \mathrm{~min}, 35 \mathrm{cycles}$ of $95^{\circ} \mathrm{C}$ for $30 \mathrm{sec}, 58^{\circ} \mathrm{C}$ for $30 \mathrm{sec}$ and $68^{\circ} \mathrm{C}$ for $30 \mathrm{sec}$. Followed by a further cycle of $68^{\circ} \mathrm{C}$ for $10 \mathrm{~min}$.

Mass spectrometry detection of gliotoxin and bismethylgliotoxin

GT uptake and BmGT conversion assays were carried out by diluting overnight cell cultures in -LEU medium to an $\mathrm{OD}_{600}=$ 0.4 and incubation at $30^{\circ} \mathrm{C}$ shaking $200 \mathrm{rpm}$ till an $\mathrm{OD}_{600}=1.2$ was reached, followed by GT $(5 \mu \mathrm{g} / \mathrm{ml})$ addition and incubation for a further $3 \mathrm{~h}$. Samples were taken every $30 \mathrm{~min}$ during the $3 \mathrm{~h}$ period, and were then centrifuged at 5,000 $\times \mathrm{g}$ to ob-

\section{REFERENCES}

1. Johnson JR, Bruce WF, Dutcher JD (1943). Gliotoxin, The Antibiotic Principle of Gliocladium fimbriatum. I. Production, Physical and Biological Properties1. Journal of the American Chemical Society 65(10): 2005-2009.

2. Gardiner DM, Waring P, Howlett BJ (2005). The epipolythiodioxopiperazine (ETP) class of fungal toxins: distribution, mode of action, functions and biosynthesis. Microbiology 151(Pt 4): 1021-1032.

3. Coleman JJ, Ghosh S, Okoli I, Mylonakis E (2011). Antifungal activity of microbial secondary metabolites. PloS one 6(9): e25321.

4. Gardiner DM, Howlett BJ (2005). Bioinformatic and expression analysis of the putative gliotoxin biosynthetic gene cluster of Aspergillus fumigatus. FEMS microbiology letters 248(2): 241-248.

5. Gardiner DM, Cozijnsen AJ, Wilson LM, Pedras MS, Howlett BJ (2004). The sirodesmin biosynthetic gene cluster of the plant pathogenic fungus Leptosphaeria maculans. Molecular microbiology 53(5): 1307-1318.

6. O'Keeffe G, Hammel S, Owens RA, Keane TM, Fitzpatrick DA, Jones $\mathrm{GW}$, Doyle S (2014). RNA-seq reveals the pan-transcriptomic impact of attenuating the gliotoxin self-protection mechanism in Aspergillus fumigatus. BMC genomics 15:894.

7. Bernardo $\mathrm{PH}$, Brasch $\mathrm{N}$, Chai $\mathrm{CL}$, Waring $\mathrm{P}$ (2003). A novel redox mechanism for the glutathione-dependent reversible uptake of a fungal toxin in cells. The Journal of biological chemistry 278(47): 46549-46555.

8. Waring P, Beaver J (1996). Gliotoxin and related epipolythiodioxopiperazines. General pharmacology 27(8): 1311-1316. tain the supernatant, which was then organically extracted using an equal volume of chloroform. Organic extracts were dried to completion under a vacuum and re-solubilized in methanol and analyzed by LC-MS (Agilent LC-MS systemModel 6340) as previously described [17].

\section{ACKNOWLEDGMENTS}

This work was funded by a Science Foundation Ireland Principal Investigator Award to SD and collaborator GWJ (PI/11/1188). GWJ has been supported by Science Foundation Ireland grant RFP08/BMT/1439. SKD is a recipient of an Irish Research Council Embark PhD Fellowship. LC-MS facilities were funded by the Irish Higher Education Authority.

\section{CONFLICT OF INTEREST}

The authors declare no existing conflicts of interest.

\section{COPYRIGHT}

(C) 2016 Smith et al. This is an open-access article released under the terms of the Creative Commons Attribution (CC BY) license, which allows the unrestricted use, distribution, and reproduction in any medium, provided the original author and source are acknowledged.

Please cite this article as: Elizabeth B. Smith, Stephen K. Dolan, David A. Fitzpatrick, Sean Doyle and Gary W. Jones (2016). Towards understanding the gliotoxin detoxification mechanism: in vivo thiomethylation protects yeast from gliotoxin cytotoxicity. Microbial Cell 3(3): 120-125. doi: 10.15698/mic2016.03.485

9. Scharf DH, Remme N, Heinekamp T, Hortschansky P, Brakhage AA, Hertweck C (2010). Transannular disulfide formation in gliotoxin biosynthesis and its role in self-resistance of the human pathogen Aspergillus fumigatus. Journal of the American Chemical Society 132(29): 10136-10141.

10. Schrettl M, Carberry S, Kavanagh K, Haas H, Jones GW, O'Brien J, Nolan A, Stephens J, Fenelon O, Doyle S (2010). Self-protection against gliotoxin--a component of the gliotoxin biosynthetic cluster, GliT, completely protects Aspergillus fumigatus against exogenous gliotoxin. PLoS pathogens 6(6): e1000952.

11. Dolan SK, O'Keeffe G, Jones GW, Doyle S (2015). Resistance is not futile: gliotoxin biosynthesis, functionality and utility. Trends in microbiology 23(7): 419-428.

12. Carberry S, Molloy E, Hammel S, O'Keeffe G, Jones GW, Kavanagh $\mathrm{K}$, Doyle S (2012). Gliotoxin effects on fungal growth: mechanisms and exploitation. Fungal genetics and biology : FG \& B 49(4): 302-312.

13. Chamilos G, Lewis RE, Lamaris GA, Albert ND, Kontoyiannis DP (2008). Genomewide screening for genes associated with gliotoxin resistance and sensitivity in Saccharomyces cerevisiae. Antimicrobial agents and chemotherapy 52(4): 1325-1329.

14. Dolan SK, Owens RA, O'Keeffe G, Hammel S, Fitzpatrick DA, Jones GW, Doyle S (2014). Regulation of nonribosomal peptide synthesis: bis-thiomethylation attenuates gliotoxin biosynthesis in Aspergillus fumigatus. Chemistry \& biology 21(8): 999-1012. 
15. Scharf DH, Habel A, Heinekamp T, Brakhage AA, Hertweck C (2014) Opposed effects of enzymatic gliotoxin $\mathrm{N}$ - and S-methylations. Journal of the American Chemical Society 136(33): 11674-11679.

16. Li B, Forseth RR, Bowers AA, Schroeder FC, Walsh CT (2012). A backup plan for self-protection: S-methylation of holomycin biosynthetic intermediates in Streptomyces clavuligerus. Chembiochem : a European journal of chemical biology 13(17): 25212526.

17. Owens RA, Hammel S, Sheridan KJ, Jones GW, Doyle S (2014). A proteomic approach to investigating gene cluster expression and secondary metabolite functionality in Aspergillus fumigatus. PloS one 9(9): e106942.
18. Owens RA, O'Keeffe G, Smith EB, Dolan SK, Hammel S, Sheridan KJ, Fitzpatrick DA, Keane TM, Jones GW, Doyle S (2015). Interplay between Gliotoxin Resistance, Secretion, and the Methyl/Methionine Cycle in Aspergillus fumigatus. Eukaryotic cell 14(9): 941-957.

19. Manzanares-Miralles L, Sarikaya-Bayram Ö, Smith EB, Dolan SK, Bayram Ö, Jones GW, Doyle S (2016) Quantitative proteomics reveals the mechanism and consequence of gliotoxin-mediated dysregulation of the methionine cycle in Aspergillus niger 10(131): 149-62.

20. Loovers HM, Guinan E, Jones GW (2007). Importance of the Hsp70 ATPase domain in yeast prion propagation. Genetics 175(2): 621-630. 